\author{
Martina Haeck
}

\title{
Behandlung von Schlafstörungen in der anthroposophischen Klinik
}

Anknüpfend an die bereits beschriebene Gruppentherapie «Nervosität» [1] möchte ich eine Einführung in die Grundlagen des Schlafes und das anthroposophische Schlafverständnis geben sowie über Schlafstörungen und deren Behandlung während eines stationären Aufenthaltes berichten.

Zusammenfassend können wir sagen, dass das gesamte stationäre Konzept unserer Klinik den gesunden Schlaf unserer Patienten unterstützt. Kommt jedoch ein Patient zu uns, bei dem die Schlafstörungen im Vordergrund stehen und ihn belasten, dann ergänzen wir dieses Konzept um die individuelle Behandlung dieses einzelnen Patienten.

Die stationäre Schlafbehandlung beginnt mit dem theoretischen Hintergrund, der Psychoedukation. Wir erklären den gesunden Schlaf, zeigen die unterschiedlichen Schlafstörungen auf und geben dem Patienten/der Patientin einen Überblick über unsere Behandlungsmöglichkeiten und die Hilfen in seinem/ihrem speziellen Fall.

\section{Zum Verständnis des Schlafes}

Unser Schlaf kann zunächst einmal als Zustand der äusseren Ruhe beschrieben werden. Er geht einher mit einer Bewusstseinsminderung, die jederzeit durch innere oder äussere Reize beendet werden kann. Genauer betrachtet ist der Schlaf jedoch ein sehr aktiver Zustand. Dies wird deutlich, wenn wir z.B. Hirnstrommessungen (EEG) im Schlaf durchführen. Her- ausgefunden wurde hier, dass es fünf verschiedene Schlafstadien gibt, die sich mehrfach im Nachtschlaf wiederholen [2]. Diese Phasen tragen ihren Namen nach den schnellen Augenbewegungen (rapid eye movement (REM)), die während unseres Schlafes auftreten oder auch nicht (Non-REM). Unser Schlaf beginnt mit den sogenannten Non-REM-Phasen in der Ein- oder Leichtschlafphase (I und II) und den Tiefschlafphasen (III und IV). Diese vier Phasen gehen einher mit einer vermehrten Ausschüttung von Hormonen und Muskelanspannung, während Herz- und Atemfrequenz, Blutdruck und Körpertemperatur vermindert sind. Im Gegensatz hierzu steht eine Puls- und Blutdruckerhöhung mit einer sehr niedrigen Muskelanspannung in der fünften Phase (REM-Phase), die ungefähr ein Viertel unserer Schlafdauer ausmacht. Im Nachtschlaf durchlaufen wir Menschen alle fünf Phasen mit unterschiedlich vielen Wiederholungen in Abhängigkeit von den unterschiedlichen Lebensphasen. Während der REM-Phasen sind wir schwer zu erwecken. Erwachen wir jedoch in dieser Phase, dann sind unsere Träume dort besonders lebhaft erinnerbar. Interessant ist auch, dass diese REM-Phase bei Kindern bis zu $9 \mathrm{~h}$ andauert und in zunehmendem Alter nur noch $2 \mathrm{~h}$ umfasst. Schauen wir uns die Schlafdauer des Menschen an, die er im Laufe seines Lebens braucht, um seinen Tag aktiv gestalten zu können, so ist diese ganz individuell und nimmt mit zunehmendem Alter ab.
Neugeborene schlafen insgesamt zwischen 12 und $20 \mathrm{~h}$ täglich, regellos über den ganzen 24-stündigen Tag verteilt. Der Schlaf ist in mehr oder weniger kurze Abschnitte geteilt, die fast nur durch die Nahrungsaufnahme unterbrochen werden. Erst allmählich werden die Perioden des nächtlichen Schlafes zusammenhängender; die Tagschlafperioden reduzieren sich, bis das Kind einen Schlafbedarf von 7-12 h hat. Erwachsene haben normalerweise eine Schlafdauer von ca. $8 \mathrm{~h}$, wobei aber grosse individuelle Unterschiede normal sind. Im Alter nimmt dann neben dem Schlafbedarf auch die Schlaftiefe ab.

Die meisten Menschen schlafen zwischen 5 und $10 \mathrm{~h}$. Es gibt die «Nachteulen», die spät müde werden, und die «Lerchen», die früh ins Bett gehen und ebenso früh aufstehen.

Warum wir überhaupt schlafen, ist noch immer nicht ausreichend erforscht. In der prähistorischen Zeit sind wir in den Phasen des Schlafes weitaus gefährdeter gewesen, unser Leben $\mathrm{zu}$ verlieren. Also muss der Schlaf demnach besondere Vorteile für das Überleben des Menschen bieten, sonst wäre er in der Evolution längst ersetzt worden.

\section{Anthroposophisches \\ Schlafverständnis}

Um das anthroposophische Schlafverständnis zu beleuchten, möchte ich auf das Modell der Viergliederung des Menschen zurückgreifen, welches die im Menschen wirkenden Kräfte er-

\section{KARGER}

(C) 2017 S. Karger GmbH, Freiburg

Fax +497614520714
Dr. med. Martina Haeck

Ärztliche Fachbereichsleiterin Psychiatrie/Psychosomatik Klinik Arlesheim

Pfeffingerweg 1, 4144 Arlesheim, Schweiz

Martina.Haeck@klinik-arlesheim.ch 
klärt [3]. Wir sprechen von den vier Wesensgliedern des Menschen - dem sichtbaren Körper (physischer Leib) und den drei übersinnlich wahrnehmbaren Wesensgliedern, d.h. dem Ätherleib als Träger der Wachstumskräfte, dem Astralleib als Träger des Seelenlebens und dem «Ich» als ein unsterblicher, geistiger Kern im Menschen. Während des Tages sind die Wesensglieder im Körper vereint und im Schlaf verlassen Seele und das «Ich» den Leib, den sie im Aufwachen wieder ergreifen. Je ausgeprägter dieser Rhythmus sich vollzieht, desto erholsamer ist unser Schlaf.

«Wenn wir abends zu Bett gehen, geraten wir in einen veränderten Bewusstseinszustand, der einige Stunden dauert. Wir sehen, hören und fühlen dabei nicht mehr bewusst, was um uns herum vorgeht. Diesen Zustand nennen wir Schlaf. Die Welt des Schlafens und des Wachens sind so verschieden, dass man sagen könnte, jeder von uns lebt in zwei Welten.» Dies beschreibt G. Löbbert im klinikeigenen Magazin «Quinte».

Den Rhythmus von Wachen und Schlafen beschreibt Fintelmann [4] als Ausgleich zwischen der abbauenden, ermüdenden Phase am Tag und dem aufbauenden, regenerierenden, erweckenden Prinzip des Schlafes. Veränderungen im Rhythmus sind immer der Ausdruck von Disharmonien, die als Tendenzen zum Kranksein gesehen werden können. "Wenn ein Mensch <aus dem Rhythmus> ist, so bedeutet dies oft, dass er seinen bisherigen Halt verloren hat. Manchmal kann es lange dauern, Wochen bis Monate, bis man seinen Rhythmus wieder gefunden hat. Daran wird erkennbar, wie wichtig Rhythmus im Leben ist», so A. Daniel ebenfalls in der «Quinte».

Je intensiver sich die höheren Wesensglieder während des Tages im Organischen einbringen können, umso besser lösen sie sich zur Nacht im Schlaf [5].

So entspricht die Therapie der Schlafstörungen einer indirekten The- rapie, indem die Verbindung der Wesensglieder mit dem Leib am Tag und die Loslösung während der Nacht harmonisiert werden. Dies kann über eine Beruhigung des Nerven-Sinnes-Systems, eine Anregung des Stoffwechselsystems oder die Stärkung des rhythmischen Systems behandelt werden und wird nachfolgend noch beschrieben.

\section{Schlafstörungen (Insomnien)}

Jeder fünfte Patient, der seinen Hausarzt konsultiert, klagt über Schlafstörungen mit Beeinträchtigung der Tagesbefindlichkeit im sozialen und beruflichen Bereich [6].

In den westlichen Industrieländern finden wir diese bei ca. 10\% der Bevölkerung [7]. Die Schlafstörungen nehmen im Alter zu, Frauen sind häufiger als Männer betroffen. In zwei Dritteln aller Fälle kommt es im Verlauf zur Chronifizierung und Einnahme von Schlaftabletten. Die Gefahr einer Gewöhnung oder auch Verschlimmerung nach Absetzen ist gegeben. Einzelne Medikamente (z.B. Benzodiazepine) erhöhen die Gefahr nächtlicher Stürze.

Backhaus et al. [6] unterteilen in primäre und sekundäre Schlafstörungen; letztere finden wir im Rahmen einer seelischen oder körperlichen Erkrankung. Eine Diagnostik zur Abgrenzung beider Arten ist natürlich die Voraussetzung für eine gute $\mathrm{Be}$ handlung des Patienten.

Aufgrund der unterschiedlich auftretenden Symptome können wir die Schlafstörungen unterscheiden in Einund Durchschlafstörungen, Früherwachen und den unerholsamen Schlaf. Störungen der Nachtruhe sind auch die Schlafunterbrechungen (Parasomnien) wie Albträume, Zähneknirschen (Bruxismus), «Zappel-Beine» (Restless Legs), Schlafwandeln, Pavor nocturnus (Aufschrecken aus dem Tiefschlaf) und Atempausen (Schlafapnoe). Sie alle können zu Beeinträchtigungen des Tagesablaufs führen.
Schlafstörungen können als die Folge und auch Wechselwirkung verschiedener Problembereiche angesehen werden. So unterscheidet Uexküll die Ursachen der Schlafstörungen aufgrund von Erkrankungen des Körpers (physisch, physiologisch) bei Schichtarbeit, Jetlag oder verlagerten Schlafphasen, psychologisch bedingt in Belastungssituationen und als Symptom bei psychiatrischen Erkrankungen [2]. Auch medikamentenbedingte Schlafstörungen (pharmakologisch) können als Ursache gesehen werden. Schlafstörungen gehen einher mit einer hohen Angespanntheit im Körper. In den Gefühlen, im Muskeltonus, im Denken und auf physiologischer Ebene bewirken sie ein Nicht-AbschaltenKönnen oder Gedankenkreisen in der Nacht.

Daraus entwickeln sich ungesunde Denkmuster (dysfunktionale Kognitionen). Wir beobachten uns ständig selbst und haben eine hohe Erwartungshaltung und grosse Angst vor den Folgen der Schlaflosigkeit. Dies wiederum kann dann zu Ängsten, depressiven Stimmungen und Tagesmüdigkeit führen; wir sind unkonzentriert und können unsere Leistung nicht mehr erbringen. Ungünstige Schlafgewohnheiten können eine Erkrankung herbeiführen, verstärken und aufrechterhalten.

\section{Behandlung auf Station}

Das gesamte stationäre Angebot unserer Psychiatrie und Psychosomatik basiert auf dem Konzept der Salutogenese. Erläutern möchte ich dies anhand der indirekten und unmittelbaren Behandlung von Schlafstörungen.

Die indirekte Unterstützung eines gesunden Schlafes geschieht durch die vertrauensbildende, wertschätzende Begegnung und Begleitung unserer Patienten in einer gesundheitsfördernden Umgebung mit ganzheitlicher Behandlung, die ich im Folgenden kurz beschreiben möchte. 
Unser erster Kontakt zu unserem Patienten ist geprägt durch unser Menschenbild: Wir sehen in ihm den aktiven Partner, der sich an seinem Genesungsprozess selbstbewusst beteiligt und bezüglich Mündigkeit und Mitbestimmung bestärkt wird. Im Praktischen sieht dies so aus, dass der gesamte stationäre Aufenthalt darauf ausgerichtet ist, gemeinsam mit dem Patienten seine Ziele zu erreichen und dies transparent mit ihm zu besprechen.

Besonders ist auch unser Krankheitsverständnis: Wir gehen in der Anthroposophischen Medizin davon aus, dass Krankheiten keine zufällig auftretenden Fehlfunktionen sind. Krankheiten werden als Prozesse angesehen, die zu körperlichen oder seelischen Störungen oder Veränderung führen, wenn die Wechselbeziehungen zwischen Körper, Geist und Seele eines Patienten nicht mehr harmonisch sind [4]. Krankheiten werden nicht pauschal, sondern individuell beurteilt oder therapiert. Ziel der Anthroposophischen Medizin ist es, die gesundheitsförderlichen Kräfte zu aktivieren, die Selbstheilungskräfte zu unterstützen und so den Krankheitsprozess günstig zu beeinflussen. Daraus ergibt sich unser Behandlungsansatz, der insbesondere dem Einbezug des Körpers grosse Beachtung schenkt. Auch die Patientenbiographie als Ausdruck einer sich ständig entwickelnden Individualität wird im therapeutischen Ansatz mit einbezogen.

Insgesamt strebt eine Behandlung neben den messbaren Befundverbesserungen - eine Steigerung des Allgemeinbefindens und eine Verbesserung der individuellen Lebenssituation des erkrankten Menschen an. Eine gesunde, auf Vertrauen beruhende Beziehung ist uns als Grundlage des therapeutischen Prozesses dabei sehr wichtig. Ausserdem legen wir grossen Wert auf ein gesundendes Milieu - sei es in der Gestaltung der Zimmer (Behaglichkeit) und Räume, der Berücksichtigung von Tagesrhythmen, aber auch im sozialen Prozess der Patientengrup- pe. Letzteres ist uns wichtig als Möglichkeit, das Ich in Gemeinschaft seinen Platz und Raum finden zu lassen.

\section{Behandlungsteam}

Das Behandlungsteam besteht neben medizinischem Fachpersonal aus Körper- und Kunsttherapeuten. Jede Patientin/jeder Patient wird durch zwei Bezugspersonen der Pflege engmaschig und individuell begleitet (Bezugspflege). Das Pflegeteam unterstützt die Patienten $24 \mathrm{~h}$ an jedem Tag. Körper- und Kunsttherapeuten haben eine spezielle Ausbildung absolviert. Der Sozialdienst arbeitet eng mit uns zusammen, sodass unser Patient sich auch in Alltagsfragen unterstützt sieht.

\section{Therapieangebote}

Ziel der Therapie ist wie eingangs beschrieben eine grösstmögliche Stabilisierung und die Anregung eines Heilimpulses. Dies wird unterstützt durch:

(1) unser Drei-Phasen-Modell für den Aufenthalt: Es bietet Sicherheit durch Strukturierung des Aufenthaltes mit Ankommen (Einhüllen im Sinne eines Vorbildes), Arbeiten (in die Eigenverantwortung kommen), Austrittsvorbereitung und Nachsorge (Transformationsphase);

(2) künstlerisches Therapiesetting: In Ergänzung zum konventionellen Therapieangebot werden künstlerische Therapien (Musiktherapie, Therapeutische Sprachgestaltung, Therapeutisches Malen und Plastizieren) sowie Heileurythmie eingesetzt;

(3) körperorientiertes Therapiesetting: In Ergänzung zum konventionellen Therapieangebot werden spezielle Pflegetherapien (Wickel, Rhythmischen Einreibungen, verschiedene Bäder und Teilbäder), Progressive Muskelrelaxation (PMR), Massagen und Physiothe- rapie eingesetzt. Lavendel; HerzSalben-Auflage; bei Schmerzen und Verspannungen Solum-ÖlAuflage;

(4) psychoedukative Gesprächsgruppen;

(5) Aktivierungsgruppe;

(6) künstlerische Erfahrung in der Gruppe (Eurythmie, Sprache, Singen, Musizieren);

(7) weitere Gruppenangebote und gemeinsame Aktivitäten;

(8) seelenhygienische Übungen wie Achtsamkeits- und Wahrnehmungsübungen, Spaziergänge, Aufmerksamkeitsübungen, Tagesrückblick usw.;

(9) spezielle Ernährung (Demeter/ Knospe) und Beratung;

(10)orale Medikamente, subkutane Injektionen, intravenöse Injektionen und Infusionen.

\section{Direkte Behandlung von Schlafstörungen}

Als Voraussetzung für die direkte Behandlung der Schlafstörungen haben wir die oben beschriebene indirekte Unterstützung aufgeführt.

Wir klären den Patienten über die optimalen Bedingungen eines guten Schlafes, die Entstehung von Schlafstörungen und deren Behandlung (Psychoedukation) auf.

Zur Verstehbarkeit als Teil der Kohärenz im Sinne der Salutogenese schauen wir uns erst das Verhalten und dann auch die Inhalte des Denkens, Fühlens und Wollens des Patienten an.

Dies geschieht, indem wir uns dann mit dem Patienten seine individuelle Tagesstruktur vor Eintritt mit Tagesund Nachtverhalten im Sinne einer Verhaltensanalyse anschauen, um die Schlafproblematik zu konkretisieren und unseren Patienten individuell therapieren zu können. Hier werden auch belastende Tagesereignisse mit einbezogen. Zur Verhaltensanalyse ist eine sorgsame, umsichtige Anamneseerhebung mit Beginn, Dauer, Verlauf 
und Beschreibung der Störung unumgänglich. Ein Schlaftagebuch im stationären Setting kann sowohl helfen zu quantifizieren als auch ungesundes Verhalten, wie z.B. Verlust der Tagesstruktur, zu identifizieren sowie Verbindung zwischen dem eigenen Verhalten und den Gefühlen herzustellen.

Das Resultat wird ausgewertet.

Beim Hinsehen können einige Dinge auffallen, z.B. zu lange Bettzeiten, $\mathrm{zu}$ frühes Schlafengehen, ein unregelmässiger Tag-Nacht-Rhythmus, Tagschlaf mit Inaktivität usw.

Individuelle Problemlösungen werden erarbeitet. Es werden dem Patienten die individuellen Werkzeuge an die Hand gegeben für den Umgang mit seinen Schlafstörungen. Durch die Handhabbarkeit erfährt der Patient eine Selbstwirksamkeit, die eine Basis schafft für Selbstvertrauen.

Die Verhaltensänderungen sind oft durch kleine Schritte erreichbar, aber Beständigkeit in der Übung neuer Verhaltensweisen ist notwendig und braucht Zeit. Dabei wird sich immer mehr die eigene Schlafkultur herausbilden. Sie beinhaltet eine richtige Vorbereitung auf den Schlaf und einen strengen Rhythmus [4]. Unsere Schlafhygiene-Regeln beinhalten:

- Wiederherstellung des Tag-NachtRhythmus;

- Übergänge zwischen Aktivität und Ruhe einplanen;

- Abstinenz von anregenden Substanzen vor dem Schlafen;

- Reduktion geistiger Tätigkeit unmittelbar vor dem Einschlafen;

- «Zubettgehen, wenn ich müde bin»;

- "Aufstehen, wenn ich wach werde»;

- Vermeidung des nächtlichen Nichtauf-die-Uhr-Schauen;

- warme Füsse;

- kein Sport oder heisse Bäder unmittelbar vor dem Schlafengehen;

- leichtes Essen vor dem Schlafen;

- schlaffördernde Atmosphäre individuell erarbeiten;

- Schlaftee oder natürliche schlaffördernde Medikamente.

Ein besonderes Augenmerk legen wir auf die Achtsamkeit. Hiermit ist die besondere Art der Aufmerksamkeitslenkung gemeint [8]. Der Patient wird angeleitet, den gegenwärtigen Augenblick absichtsvoll und nicht wertend wahrzunehmen. Spezielle Übungen dienen als Gegenpol zur halbautomatisierten Informationsverarbeitung. Ziel ist, das anzunehmen, was jetzt geschieht, und eine Ausgewogenheit zu finden zwischen Akzep$\tan z$ und Veränderung. Nicht die Inhalte der Gedanken werden verändert, sondern die Haltung hierzu. Achtsamkeitsübungen, die wir mit den Patienten einüben, sind:

Pflanzenbetrachtungen: Anschauen und Beschreibung einer Pflanze und deren Veränderung wahrnehmen während des gesamten Aufenthaltes.

Tastübungen: Mit geschlossenen Augen einen Gegenstand beschreiben und ihn erkennen.

Achtsames Gehen: Konzentration auf die Tätigkeit des Schreitens mit Wahrnehmung der einzelnen Phasen.

Barfusspfad: Unterschiedliche Reize (z.B. Sand, Stein, Erde, Rindenmulch) auf dem Boden wahrnehmen.

Atemübungen: Bewusstes Ein- und Ausatmen verknüpft mit angenehmen, hilfreichen Bildern.

Labyrinth: Achtsames Schreiten durch unser Labyrinth und Wahrnehmen des Öffnen und Schliessen des Kreises als Sinnbild für den Lauf des Lebens.

Als Einschlafhilfen werden vor allem die Übergänge zum Schlaf bewusst gestaltet. Die Abendruhe wird eingeleitet mit einem Tagesrückblick. In diesem geht man in Gedanken rückwärts durch den Tag, vom Abend bis zum Morgen, indem man sich einzelne Stationen und Situationen bildhaft ins Gedächtnis ruft. Man beginnt mit wenigen Situationen und steigert sich mit der Zeit. Gut ist, sich auf Wesentliches $\mathrm{zu}$ konzentrieren und sich nicht in Details zu verlieren. Insgesamt sollte dieser Tagesrückblick 5 min dauern.

Einzelne Patienten legen sehr gerne ein Sonnen-Tagebuch an, in dem gute Momente des Tages aufgezählt werden als Brücke in die Nacht. Die Wahrnehmung wird gelenkt, dass auch in einem vermeintlich schlechten Tag das Gute gesehen und angenommen werden kann. Besonders angenehm sind schlaffördernde Einreibungen und Auflagen, z.B. mit Lavendel, die den Körper in die Ruhe und Nachtschwere kommen lassen. Manche Patienten können beruhigter schlafen, wenn die Nachtwache ein oder zwei Mal während der Nacht im Zimmer vorbeischaut.

Ist der Schlaf unterbrochen, dann hilft das Aufstehen mehr, als sich wach im Bett zu wälzen. Ein warmer Tee kann hier hilfreich sein. Ein Ausweg aus den nächtlichen Grübelschleifen ist das Formenzeichnen. Die Konzentration auf diese fliessende Tätigkeit bringt Erleichterung. Die Spirale aus Anspannung und Schlaflosigkeit wird unterbrochen.

Der begleitete Gedankenstopp mit alternativen Gedankengängen wird von unseren Patienten ebenfalls als sehr hilfreich empfunden.

Gegen Ende der Nacht unterstützen wir dann bei einzelnen Patienten die Aufwachphase mit Wickeln oder Auflagen, um die «Angst vor dem Tag» zu nehmen.

Wir helfen unseren Patienten, in stationärer Behandlung wieder einen gesunden Rhythmus zu finden. Im anthroposophischen Sinne ist das die Pflege des Ätherischen. Ziel ist die Harmonisierung bestehender und Aufbau neuer Rhythmen mit bewusster Gestaltung der einzelnen Phasen und Pflege der Übergänge [9]. Dazu wird Struktur entwickelt im Sinne einer Rhythmisierung von Abläufen mit (ab-)wechselnden Phasen der Aktivität und der Ruhe, des Empfangens und der Selbständigkeit, von Sprechen und Hören, von Gemeinschaft und Alleinsein (eigenem Gestalten).

Rhythmen, die wir gestalten können: - Tagesrhythmus: Bei der Strukturierung des Tages helfen Tagesaktivität, Einhalten der Abend- und Nachtruhe, Tagesrückblick, Sonnen-Tagebuch, Einbau von Entspannungstechniken wie PMR oder 
autogenes Training, Schlafrestriktion (gefühlte Schlafdauer als Ausgangswert), regelmässig ins Bett gehen und regelmässig aufstehen.

- Wochenrhythmus: Ein Sieben-Tage-Rhythmus, wobei ein Tag be-

\section{Literatur}

1 Haas H: Salutogenese und achtsamkeitsorientierte anthroposophische Psychotherapie - am Beispiel ambulanter Gruppentherapien bei „Nervosität“. Schweiz Z Ganzheitsmed 2017; 29:DOI: 10.1159/000484462.

2 Köhle K, Herzog W, Joraschky P, et al. (Hrsg): Uexküll, Psychosomatische Medizin, ed 8. München, Elsevier, 2016.

3 Steiner R: Vierter Vortrag, Berlin, 10. Dezember 1912, aus „Das Leben zwischen Tod und neuer Geburt im Verhältnis zu den kosmischen Tatsachen“, GA 141, Dornach, Rudolf Steiner, 1983. wusst anders gestaltet ist. Diesen Tag nach Lust und Laune gestalten. An diesem Tag kann der psychophysische Organismus seinen eigenen Rhythmus wiederfinden.

4 Fintelmann V: Intuitive Medizin, ed 6. Bern, Haug, 2016.

5 Roemer F: Therapiekonzepte der anthroposophischen Medizin: Stufenpläne mit Differenzialdiagnostik. Bern, Haug, 2014.

6 Backhaus J, Junghanns K, Mueller-Popkes K, Broocks A, Riemann D, Hajak G, Hohagen F: Short-term training increases diagnostic and treatment rate for insomnia in general practice. Eur Arch Psychiatry Clin Neurosci 2002;252:99-104.
- Monatsrhythmus: Viele Menschen spüren die Mondphasen; er kann eine besondere Wachheit bringen.

- Jahresrhythmus: Beim Feiern der Jahreszeiten und ihrer Feste sind unsere Patienten mit beteiligt.

7 Lieb K, Frauenknecht S (Hrsg): Intensivkurs Psychiatrie und Psychotherapie, ed 8. München, Elsevier, 2015.

8 Michalak J, Heidenreich T, Williams JMG: Achtsamkeit, ed 1. Fortschritte der Psychotherapie - Band 48. Göttingen, Hogrefe, 2012.

9 Dahlem H, Hölzer-Hasselberg R, Schopper C, Steinmann-Lindner S: Burnout - mit der Krise wachsen. Berlin, Salumed, 2011. 